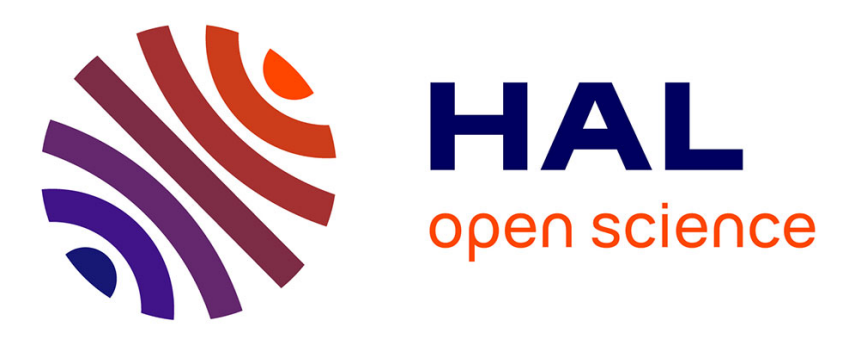

\title{
Elastic Signature of Flow Events in Supercooled Liquids Under Shear
}

Joyjit Chattoraj, Anael Lemaitre

\section{To cite this version:}

Joyjit Chattoraj, Anael Lemaitre. Elastic Signature of Flow Events in Supercooled Liquids Under Shear. Physical Review Letters, 2013, 111 (6), pp.066001-066001-5. 10.1103/PHYSREVLETT.111.066001 . hal-00969131

\section{HAL Id: hal-00969131 https://hal.science/hal-00969131}

Submitted on 10 Apr 2014

HAL is a multi-disciplinary open access archive for the deposit and dissemination of scientific research documents, whether they are published or not. The documents may come from teaching and research institutions in France or abroad, or from public or private research centers.
L'archive ouverte pluridisciplinaire HAL, est destinée au dépôt et à la diffusion de documents scientifiques de niveau recherche, publiés ou non, émanant des établissements d'enseignement et de recherche français ou étrangers, des laboratoires publics ou privés. 


\title{
Elastic signature of flow events in supercooled liquids under shear
}

\author{
Joyjit Chattoraj ${ }^{1,2}$ and Anaël Lemaître ${ }^{1}$ \\ 1) Université Paris-Est, Laboratoire Navier (UMR 8205), \\ CNRS, ENPC, IFSTTAR, F-77420 Marne-la-Valle, France and \\ 2) Institute of Physical Chemistry, University of Münster, Corrensstrasse 30, D-48149 Münster, Germany
}

(Dated: August 19, 2013)

\begin{abstract}
Using numerical simulation of a 2D Lennard-Jones system, we study the cross-over from shearthinning to Newtonian flow. We find the short-time elastic response of our system essentially unchanged through this cross-over, and show that, in the Newtonian regime, thermal activation triggers shear transformations, i.e. local irreversible shear events that produce Eshelby (long-ranged, anisotropic) deformation fields as previously seen in low- $T$ glasses. Quite surprisingly, these Eshelby fields are found to persist much beyond the $\alpha$-relaxation time, and shear-thinning to coincide with the emergence of correlations between shear relaxation centers.
\end{abstract}

It is now well documented that, in glassy solids, plastic deformation results from the accumulation of "shear transformations" occuring within "zones" a few particle wide [1]. These rearrangements give rise, in the surrounding (isotropic) elastic medium, to "Eshelby" stress changes [2], which are anisotropic - with $\cos (4 \theta)$ symmetry in $2 \mathrm{D}$ - by virtue of the tensorial character of the shear sources. Eshelby fields bias the occurrence probabilities of plastic events depending on their locations relative to prior ones and, at low temperature, when strain sources are predominantly oriented (in a tensorial sense) along external deformation, promote avalanche behavior, i.e. the organization of plastic events along bandslike patterns [3, 4]. Studies of stress [5] and diffusion [6] in systems sheared at finite temperature (T) have evidenced that avalanche dynamics remain at work up to $T \lesssim 0.75 T_{g}$, with $T_{g}$, the glass transition temperature.

Knowledge, however, is still fragmentary as to how deformation mechanisms change around $T_{g}$. The response of a supercooled liquid is Newtonian (steady shear stress $\sigma \propto$ strain rate $\dot{\gamma}$ ), at high $T$ and low $\dot{\gamma}$; it progressively becomes shear-thinning ( $\sigma$ grows sublinearly in $\dot{\gamma}$ ), when decreasing $T$ /increasing $\dot{\gamma}$ [7]. Furukawa et al [8], have found the spatial correlation of the displacement field to be anisotropic in the shear-thinning regime but not in Newtonian flows. Since the low- $T$ flips create anisotropic deformation fields we must ask whether this observation signals a change in the very nature of elementary relaxation events, or a change of correlation properties between them. This question is crucial to any theoretical approach to the rheology of supercooled liquids.

To characterize flow events at both high and low temperatures, we will focus on the shear strain field and its spatial auto-correlation, an approach apparented to recent studies of avalanche dynamics in numerical simulations [9] and of the transition to shear banding in experiments [10]. Borrowing from recent advances in diffuse wave acoustics [11] we show that the auto-correlation of the short time (non-affine) strain field captures the $d y$ namic elastic Green function of the material; this enables us to show that the longitudinal and transverse sound speeds are unchanged through the shear-thinningto-Newtonian cross-over. Using direct measurements of elastic moduli in inherent states (IS's) we then find that the high frequency elastic properties of our system are essentially $T$-independent and very weakly $\dot{\gamma}$-dependent up into the Newtonian regime. From long-time strain autocorrelation data we next bring evidence that, in the Newtonian regime, thermal fluctuations trigger irreversible shear transformations giving rise to (anisotropic) Eshelby fields, like in low- $T$ glasses; quite strikingly, these fields are found to persist much beyond the $\alpha$ relaxation time.

Our work thus substantiates the idea that supercooled liquids are "solids that flow" [12], and meanwhile reveals two major surprises: (i) Eshelby strains are observed around and above $T_{g}$, in the Newtonian regime - when they are usually thought to be peculiar to low$T$, plastic, deformation; (ii) correlations between shear relaxation centers emerge as soon as the system enters, for $T$ decreasing, into the shear-thinning regime. The Newtonian-to-shear-thinning cross-over thus appears to be controlled by the competition between the flow events needed to relax shear and randomly oriented shear events triggered by thermal activation.

Simulations are performed using the same 2D binary LJ mixture as in [4-6]. From athermal simulations [4] the bulk and shear moduli are: $K \simeq 85, \mu \simeq 25$. The mass density $\rho \simeq 1.77$, whence p- and s-wave speeds (in 2D) $c_{l}=\sqrt{(K+\mu) / \rho} \simeq 7.9$ and $c_{t}=\sqrt{\mu / \rho} \simeq 3.8$. (We use throughout reduced LJ units). From the decay of the incoherent scattering function, we find $\tau_{\alpha}=10^{4}$ at $T=T_{g} \sim 0.27$. Simple shear is implemented using LeesEdwards boundary conditions with flow lines along the $x$ axis. Only steady state data are considered for each parameter set (linear system size $L, \dot{\gamma}$, and $T$ ). Plots of viscosity $\eta=\sigma / \dot{\gamma}$ (with $\sigma$ the steady shear stress) vs $\dot{\gamma}$ are presented on Fig. 1 for different $T$ 's, using $L=40$, a size beyond which $\eta$ is size-independent [5]. We find the usual cross-over from a Newtonian plateau at low $\dot{\gamma}$ and high $T$, to shear-thinning. For $T \leq 0.2$, plastic flow is known to be governed by the same mechanisms - zone flips and avalanche behavior - as athermal systems $[5,6]$.

To construct a coarse-grained non-affine strain field, $\underline{\underline{\epsilon}}(\underline{r} ; t, t+\Delta t)$, between times $t$ and $t+\Delta t$, we follow the method proposed in [13], using the normalized coarsegraining function: $\phi(\underline{r}) \propto 1-2\left(r / r_{c}\right)^{2}+\left(r / r_{c}\right)^{8}$ for $r<r_{c}$, 

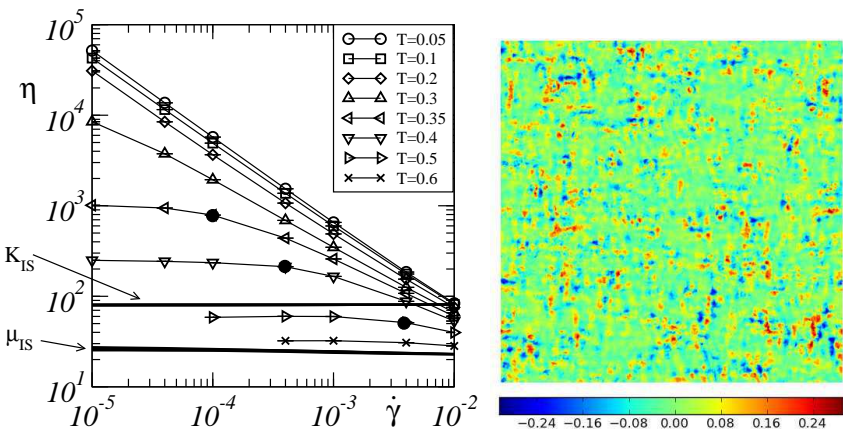

FIG. 1. Left: Steady flow viscosity $\eta=\sigma / \dot{\gamma}$ vs. $\dot{\gamma}$ for different T's; bullets: $\left(\dot{\gamma}_{c}(T), \eta\left(\dot{\gamma}_{c}(T)\right)\right.$ with $\dot{\gamma}_{c}(T)$ from [6]; thick lines: $K_{\text {IS }}$ and $\mu_{\text {IS }}$ (see text). Right: A typical strain map (color online), using $L=160, T=0.35, \dot{\gamma}=10^{-4}, \Delta t=100, r_{c}=2$.

$\phi(\underline{r})=0$ otherwise [14]. A typical map of the shear strain $\epsilon_{x y}$ is shown on Fig. 1-(right) for $r_{c}=2$ : regions where inelastic events have occurred exhibit large strain values, which contrast against the background of small elastic fluctuations. We have systematically used different coarse-graining scales $r_{c}=1,2$, and 4 , to check for consistency, and found similar conclusions; in the rest of this paper, we take $r_{c}=1$, which provides better statistics.

On Fig. 2, plots of the space and ensemble average $\left\langle\epsilon_{x y}^{2}\right\rangle$ vs. $\Delta t$ present the usual sequence of convective, caging and diffusive regimes. For all of our T's and $\dot{\gamma}$ 's, $\left\langle\epsilon_{x y}^{2}\right\rangle / T$ [see Fig.2-(a)] collapse at short times: strain fluctuations are then $\propto T$, i.e. result primarily from fast thermal vibrations around IS's. At long times, $\left\langle\epsilon_{x y}^{2}\right\rangle / \Delta t$ reaches a plateau, which defines a diffusion coefficient $D_{x y}=\lim _{\Delta t \rightarrow \infty}\left\langle\epsilon_{x y}^{2}\right\rangle / \Delta t$. Plots of $D_{x y}$ vs $T$ for different $\dot{\gamma}$ [Fig. 2-(right)] show the same features as a similar display of transverse particle diffusion data [6]: (i) for $T \gtrsim T_{g}, D_{x y}(T ; \dot{\gamma})$ matches at low $\dot{\gamma}$ 's its value $D_{x y}^{\text {eq }}(T)$ in the unsheared equilibrated supercooled liquid (thick solid line); (ii) $D_{x y}(T ; \dot{\gamma})$ deviates from $D_{x y}^{\mathrm{eq}}(T)$ at low $T$, high $\dot{\gamma}$, thus marking the entry in a regime where strain diffusion is primarily due to plastic events. The crossover between the "temperature-controlled" and "straincontrolled" regimes can be roughly located by identifying where the $D_{x y}(T ; \dot{\gamma})$ curves merge onto the equilibrium line $D_{x y}^{\mathrm{eq}}(T)$. The resulting $\dot{\gamma}_{c}(T)$ values (not shown) are consistent with those identified in [6] from diffusion data. On the viscosity plot of Fig. 1-(left), we place bullets at the $\left(\dot{\gamma}_{c}(T), \eta\left(\dot{\gamma}_{c}(T)\right)\right)$ points to show that they also mark the merging of $\eta(\dot{\gamma})$ into the low- $\dot{\gamma}$ Newtonian plateaus.

We concentrate in the following, on the spatial autocorrelation of the accumulated strain field: $C_{\alpha \beta \kappa \chi}(\underline{R} ; \Delta t)=$ $\left\langle\epsilon_{\alpha \beta}(\underline{r} ; t, t+\Delta t) \epsilon_{\kappa \chi}(\underline{r}+\underline{R} ; t, t+\Delta t)\right\rangle$, and start by considering its short-time dynamics. For this purpose, we adapt in [14] an argument by Lobkis and Weaver which establish a fluctuation theorem for the dynamic response of elastic systems [11]. Namely, we show that if particle motion subsumes to harmonic thermal vibrations around IS's: $\underline{\underline{C}}(\underline{R} ; \Delta t)=T \underline{\underline{G}}^{*}(\underline{R} ; \Delta t)$, with $\underline{\underline{G}}^{*}(\underline{R} ; t)$ the ensemble-averaged dynamic strain-strain Green function.
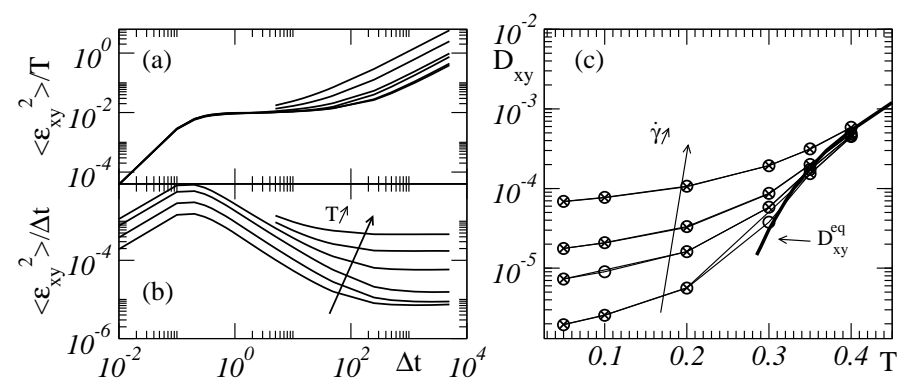

FIG. 2. Left: $\left\langle\epsilon_{x y}^{2}\right\rangle / T$ (a) and $\left\langle\epsilon_{x y}^{2}\right\rangle / \Delta t$ (b) vs $\Delta t$, for $T=$ $0.05,0.1,0.2,0.3,0.35,0.4$, and $\dot{\gamma}=4.10^{-5}$. (c): symbols: $D_{x y}$ vs. $T$ for $\dot{\gamma}=10^{-5}, 4.10^{-5}, 10^{-4}, 4.10^{-4}, 10^{-3}, 4.10^{-3}$, $10^{-2}$ and both $L=80$ (circles) and 160 (triangles); thick solid line: $D_{x y}^{\mathrm{eq}}$ in the equilibrated supercooled liquid with $L=80$.

2D plots of $C_{x y x y}$ (as of now, abbreviated as $C_{x y}$ ) at short times are displayed on Fig. 3: as per above, they reveal the strain response to a stress dipole [15] applied at the origin at $t>0$, and accordingly show two wave fronts propagating away from the origin at (roughly) the velocities $c_{l}$ and $c_{t}$ which are estimated using athermal simulation data [16]. Quite remarkably, the front velocities match their $T=0$ estimates, even though we use here $T=0.3$ and $\dot{\gamma}=10^{-5}$, which sets our system well inside the Newtonian regime. Cuts of $C_{x y}$ along the $x$ axis, on Fig. 3 (bottom) [17], emphasize that the transverse sound speed is insensitive to $T$ over all of our temperature range, which extends beyond $T_{g}$.
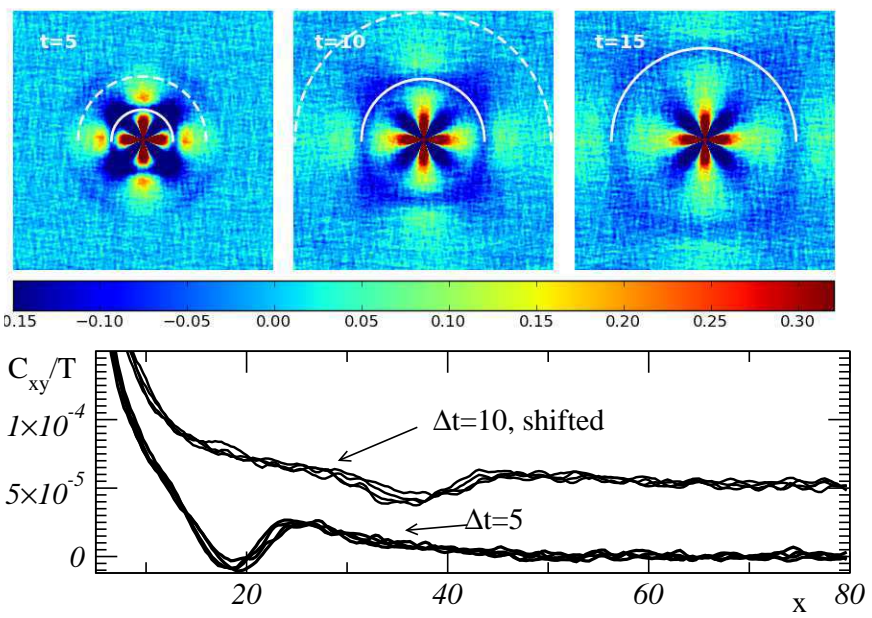

FIG. 3. (Color online): Top: $10^{4} C_{x y}(\underline{R} ; \Delta t) / T$ for $\underline{R} \in$ $[-L / 2, L / 2]^{2}(L=160), T=0.3, \dot{\gamma}=10^{-5}, r_{c}=1$, times $\Delta t=5,10$, and 15 (from left to right). White semicircles have radii $\Delta t c_{t}$ (solid) and $\Delta t c_{l}$ (dashed). Bottom: $C_{x y}\left(x \underline{e}_{x} ; \Delta t\right) / T$ vs $x$ at $\Delta t=5$ and 10 (the latter shifted by $510^{-5}$ ) for $\dot{\gamma}=10^{-5}, T=0.05,0.1,0.2$, and 0.3 .

Beyond $T=0.3$, the caging regime is too short to probe elasticity using this method. We have thus also measured the elastic moduli of IS's (using [18]) sampled from steady flow configurations. $K_{\mathrm{IS}}$ and $\mu_{\mathrm{IS}}$ are reported on the viscosity plot, Fig. 1-(left), for all our $\dot{\gamma}$ 's 

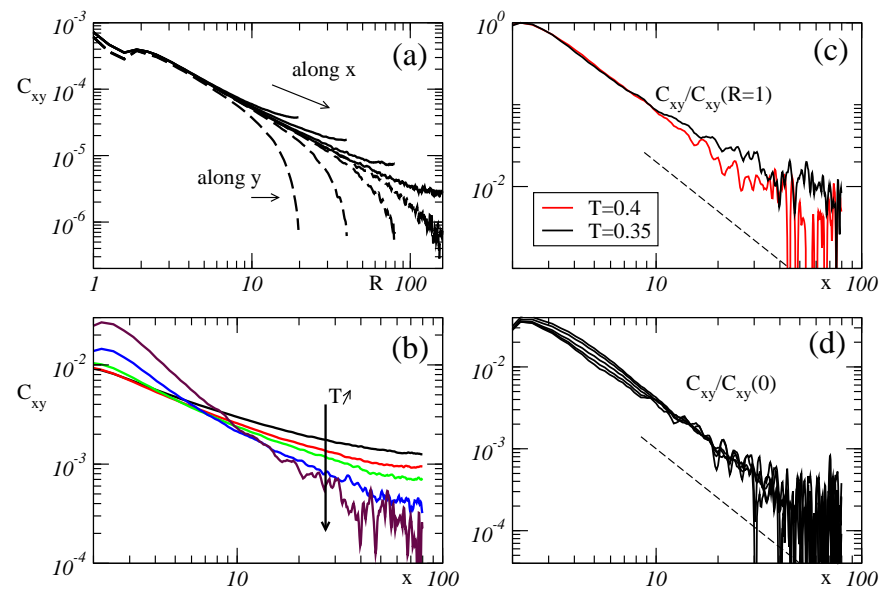

FIG. 4. (Color online) (a) $C_{x y}$ vs distance $R=x$ or $y$ along both the $x$ and $y$ axes for $\dot{\gamma}=10^{-4}, \Delta t=100, T=0.1$, $L=40,80,160$, and $320 ;(\mathrm{b}, \mathrm{c}, \mathrm{d})$ present only cuts along $x$; (b) $\dot{\gamma}=10^{-4}, \Delta t=2.10^{3}, L=160, T=0.05,0.1,0.2,0.3$, 0.35 ; (c) $C_{x y} / C_{x y}(R=1)$, same parameters, but $\mathrm{T}=0.35$ and 0.4 ; (d) $C_{x y} / C_{x y}(0)$, for $\dot{\gamma}=10^{-5}, T=0.35$, and $\Delta \gamma=1 \%$, $2 \%, 5 \%, 10 \%$, and $20 \%$, i.e. time intervals $\Delta t=10^{3}, 2.10^{3}$, $5.10^{3}, 10^{4}$, and $2.10^{4}$. Thin dashed lines: slope -2 .

and T's up to 0.4. The near-constancy of elastic moduli sharply contrasts with the order-of-magnitude changes in viscosity [19]. We thus find that, through the shearthinning-to-Newtonian cross-over, the short-time elastic properties of our sheared systems are only marginaly sensitive to temperature and strain-rate.

If the elastic properties of the IS are similar on either side of the crossover, then shouldn't the relaxation events occurring in the the liquid couple with the elastic matrix, like low- $T$ flips, and emit acoustic signals carrying, at least for short times, Eshelby strain fluctuations? What kind of perturbation are these events thus creating in the liquid? To answer this crucial question we now examine the non-affine strain field accumulated over long times, in the diffusive regime of strain fluctuations.

Beyond the caging regime, $\epsilon_{x y}(t, t+\Delta t)$ compounds thermal strain fluctuations with the accumulating plastic deformation. Denoting $\epsilon_{x y}^{\mathrm{pl}}(t, t+\Delta t)$ the strain associated with the change of IS between $t$ and $t+\Delta t$, and $\epsilon_{x y}^{\mathrm{el}}(t)$ that associated with the departure of each instantaneous configuration from its IS, we have: $\epsilon_{x y}(t, t+\Delta t)=$ $-\epsilon_{x y}^{\mathrm{el}}(t)+\epsilon_{x y}^{\mathrm{pl}}(t, t+\Delta t)+\epsilon_{x y}^{\mathrm{el}}(t+\Delta t)$. The short-time autocorrelation of the strain field, which we studied above, is nothing but the autocorrelation of $\epsilon_{x y}^{\mathrm{el}}(t+\Delta t)-\epsilon_{x y}^{\mathrm{el}}(t)$. Moreover, because vibration modes have uncorrelated phases, the autocorrelation of any field $\epsilon_{x y}^{\mathrm{el}}(t)$ alone is zero $[11,14]$. At long times, hence, as hopping events accumulate, the autocorrelation of $\epsilon_{x y}^{\mathrm{el}}(t+\Delta t)-\epsilon_{x y}^{\mathrm{el}}(t)$ must vanish and then only remains in $C_{x y}(\underline{R} ; \Delta t)$ the contribution of $\epsilon_{x y}^{\mathrm{pl}}$.

Before proceeding, our discussion has to address one technical difficulty: as shown on Fig. 4-(a), typical cuts of $C_{x y}(\underline{R} ; \Delta t)$, at low temperature, present a strong $x, y$ asymmetry. Clearly, this is a finite size effect arising from the Lees-Edwards boundary conditions [14]. As system size increases, however, the $C_{x y}$ values along both the $x$ and $y$ axes noticeably collapse on the same master curve; in particular, we see that $C_{x y}$ cuts along $x$, for $L=160$, provide a meaningful approximation for the large $L$ autocorrelation data, up to significant distances $\simeq 40$. We focus of such cuts in the next plots.

Correlation data for $\dot{\gamma}=10^{-4}$, in the diffusive regime of $\left\langle\epsilon_{x y}^{2}\right\rangle$ (namely, for $\Delta t=2.10^{3}$ ) are presented both as cuts on Fig. 4-(b-c) and as 2D plots on Fig. 5. As discussed above, the conspicuous $x, y$ asymmetry on the low- $T$ 2D plots is a finite size effect. Fig. 4-(b) shows that the correlation tails decrease slowly in the regime where avalanche behavior is expected to be at work, up to $T=0.2[5,6]$, and more sharply between $T=0.2$, and 0.35 . The decay of correlations with increasing $T$ is accompanied, on 2D plots, by a progressive emergence of the $\cos (4 \theta)$ symmetry. A decrease of the correlation tail can still be seen between $T=0.35$ and $T=0.4$ on Fig 4(c). At this latter temperature, the correlation function present both a $1 / R^{2}$ decay and $\cos (4 \theta)$ symmetry.

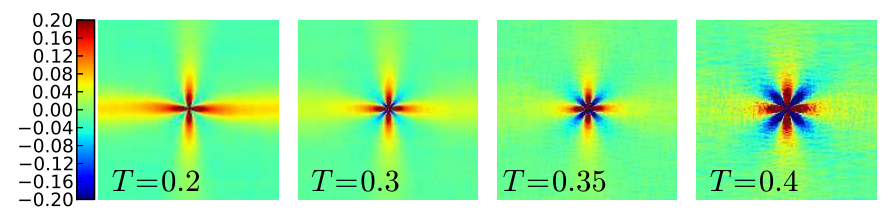

FIG. 5. 2D plots of $10^{2} C_{x y}(\underline{R})$ at $\Delta t=2.10^{3}, \dot{\gamma}=10^{-4}$, $T=0.2,0.3,0.35,0.4$ (from left to right).

It is striking here to find non-vanishing anisotropic correlations of the strain field at $T=0.4$, a very high temperature, much above $T_{g}$. We observe the same feature for all our $(\dot{\gamma}, T)$ 's in the Newtonian regime: namely $C_{x y} \propto \cos (4 \theta) / R^{2}$, the same analytic form as the far-field strain (or stress) produced by an Eshelby event [20]. To interpret this observation, let us write the strain accumulated between $t$ and $t+\Delta t, \epsilon_{x y}=\sum_{e} \epsilon_{x y}^{e}$, as the sum of the contributions of individual events, and note that $C_{x y}(\underline{R} ; \Delta t) / C_{x y}(\underline{0} ; \Delta t)=\left\langle\epsilon_{x y}^{e}(\underline{r}) \epsilon_{x y}^{e}(\underline{r}+\right.$ $\underline{R})\rangle /\left\langle\left(\epsilon_{x y}^{e}\right)^{2}\right\rangle$ when events are independent. Decomposing each $\epsilon_{x y}^{e}$ into the large but localized contribution of the relaxing center, and the order-of-magnitude smaller, spatially extended, elastic strain field, $\epsilon_{x y}^{e}=\epsilon_{x y}^{\text {core }}+\epsilon_{x y}^{\text {field }}$, we now see that $C_{x y}(\underline{R} ; \Delta t) / C_{x y}(\underline{0} ; \Delta t) \propto\left\langle\epsilon_{x y}^{\text {core }}(\underline{r}) \epsilon_{x y}^{\text {core }}(\underline{r}+\right.$ $\underline{R})\rangle+2\left\langle\epsilon_{x y}^{\text {core }}(\underline{r}) \epsilon_{x y}^{\text {field }}(\underline{r}+\underline{R})\right\rangle$. The first term on the rhs captures details of particle motion inside relaxation centers; it must hence quickly vanish with distance and become negligible beyond length scales $\sim$ a zone size: only the second term remains in the far-field and hence $C_{x y}(\underline{R} ; \Delta t) / C_{x y}(\underline{0} ; \Delta t)$ capture the average strain field produced by an irreversible event [21].

On this basis, we interpret the $\cos (4 \theta) / R^{2}$ form of the correlation function in the Newtonian regime as signaling that strain fluctuations result from the accumulation of independent Eshelby events. Since we learned from strain 
diffusion that, in this regime, strain fluctuations are also identical to those of the equilibrated supercooled liquid, we now conclude that in supercooled liquids, thermal fluctuations activate independent shear transformations, i.e. local shear events, which couple elastically with the embedding medium and give rise to Eshelby strains.

The idea that relaxation events produce Eshelby fields in supercooled liquids seems quite contrary to current expectations. So, to further assess the degree of persistence of these long-ranged elastic effects, we provide on Fig. 4-(d), $C_{x y}(\underline{R} ; \Delta t) / C_{x y}(\underline{0} ; \Delta t)$ data for $T=0.35$ and $\dot{\gamma}=10^{-5}$, in the Newtonian flow, at increasing time intervals, up to $\Delta t=2.10^{4}$. This latter value is much larger than our estimate of $\tau_{\alpha}(0.35) \sim 100$ [6] based on the usual analysis of the intermediate scattering function; over this time interval, $\left\langle\epsilon_{x y}^{2}(\Delta t)\right\rangle$ increases $10^{3}$-fold from the caging plateau. The curve collapse entails that the Eshelby strains created in the supercooled liquid by irreversible events are not erased by later ones but persist and accumulate diffusively at timescales much beyond $\tau_{\alpha}$. The observation of lasting Eshelby fields in a Newtonian liquid is the primary outcome of our work.

We now know that the Newtonian flow proceeds by the accumulation of Eshelby events which have similar features to the low- $T$ plastic ones. Moreover, we observed on Fig. 5 that the signature of avalanche behavior at low- $T$ is not the $x, y$ asymmetry (which is just a finite size effect): it is instead the imbalance between positive and negative lobes, which reflects the preferential alignement of shear events along both the $x$ and $y$ axes. With this at hand, let us now come back to the comparison of $T=0.35$ and $T=0.4, \dot{\gamma}=10^{-4}$, data: these two temperatures were chosen because [see Fig. 1-(left)] the system lies in the middle of the cross-over at $\dot{\gamma}=10^{-4}, T=0.35$, and inside the Newtonian regime for $T=0.4$. The slower than $1 / R^{2}$ decay of $C_{x y}$ at $T=0.35$ associated with the slight imbalance between \pm lobes on the corresponding $2 \mathrm{D}$ plot now shows that the entry (with decreasing $T$ ) inside the shear-thinning regime is associated with the emergence of correlations between shear relaxation events. This is the second important result of our work.

The following picture is now unfolding. Measurements of sound propagation and of elastic moduli in IS show that the elastic properties of sheared supercooled liquids, i.e. the local curvatures of the potential energy landscape, are essentially statistically unchanged when crossing over from the glassy to the shear-thinning and up into the Newtonian response, a result which extends to sheared systems recent findings in equilibrated ones [22]. At the time scale relevant to the unfolding of shear transformations, high and low $T$ systems are hence statistically identical. The first important outcome of our work is the direct evidence that in supercooled liquids thermal activation triggers local shear events which create longranged, and lasting, Eshelby fields. The second, that the emergence of correlations between shear relaxation events with decreasing $T$ coincides with the Newtonian to shear-thinning cross-over. Clearly, these correlations will have to be taken into account in future theories of shear-thinning behaviour.

The striking persistence of Eshelby fields entails that the strain changes due to irreversible events simply add up, up to very large times. Of course, this result is here obtained using 2D Lennard-Jones system with periodic boundary conditions, and up to moderate deformations that permit a small strain (geometrically linear) analysis. Pending future studies, possibly in 3D, using complex loading paths that can probe finite deformation effects, we tentatively interpret our observation as follows. In undercooled liquids, where the Newtonian-to-shearthinning crossover is found, irreversible events are expected to be local [23], i.e. to involve a change of reference state in small regions. As explained by Eshelby, such changes amount to introducing localized forces acting on the underlying elastic structure (inherent states). As time proceeds, the total deformation is hence that due to the accumulation of all these force sources. Additivity follows if these events are unlikely to unfold at the same time and place - a not so stringent condition which is expected to be fullfilled at least in supercooled liquids, where irreversible processes are activated.

The authors are grateful to Christiane Caroli, whose sharp insights, critiques and comments have been of precious help at many stages in the elaboration of this work. We were granted access to the HPC resources of IDRIS under the allocation 2010-99644 by GENCI (Grand Equipement National de Calcul Intensif).
[1] C. E. Maloney and A. Lemaître, Phys. Rev. E 74, 016118 (2006).

[2] G. Picard, A. Ajdari, F. Lequeux, and L. Bocquet, Eur. Phys. J. E 15, 371 (2004).

[3] C. Maloney and A. Lemaître, Phys. Rev. Lett. 93, 016001 (2004).

[4] A. Lemaitre and C. Caroli, Phys. Rev. Lett. 103, 065501 (2009).

[5] J. Chattoraj, C. Caroli, and A. Lemaître, Phys. Rev. Lett. 105, 266001 (2010).

[6] J. Chattoraj, C. Caroli, and A. Lemaitre, Phys. Rev. E
84, 011501 (Jul. 2011).

[7] M. Fuchs and M. Ballauff, J. Chem. Phys. 122, 094707 (Mar. 2005).

[8] A. Furukawa, K. Kim, S. Saito, and H. Tanaka, Phys. Rev. Lett. 102, 016001 (2009).

[9] C. E. Maloney and M. O. Robbins, J. Phys.: Cond. Mat. 20, 244128 (Jun. 2008).

[10] V. Chikkadi, G. Wegdam, D. Bonn, B. Nienhuis, and P. Schall, Phys. Rev. Lett. 107, 198303 (Nov. 2011).

[11] O. I. Lobkis and R. L. Weaver, J. Acoust. Soc. Amer. 110, 3011 (Dec. 2001). 
[12] J. C. Dyre, Phys. Rev. E 76, 041508 (Oct. 2007).

[13] I. Goldhirsch and C. Goldenberg, Eur. Phys. J. E 9, 245 (2002), ISSN 1292-8941, 1292-895X.

[14] See Supplementary material at ............. for technical details.

[15] W. Slaughter, The Linearized Theory of Elasticity, 1st ed. (Birkhäuser, Boston, 2001).

[16] Note that in an elastic continuum, the dynamic response to a pure shear source presents only the transverse front: our observation of both fronts entails that scattering on the elastic fluctuations by structural disorder couples shear and longitudinal waves.

[17] The broadening of the fronts is characteristic of wave propagation in $2 \mathrm{D}$, but arises also partly from scattering.

[18] A. Lemaître and C. Maloney, J. Stat. Phys. 123, 415 (2006).

[19] Over the studied parameter range, the relative changes of $K_{\text {IS }}$ are $\lesssim 2 \%$; at fixed $T, \mu_{\text {IS }}$ decreases somewhat $\sim 10 \%$ with $\dot{\gamma}$; at any fixed $\dot{\gamma}$, it increases very slightly $(\sim 5 \%)$ with $T$, a result consistent with [22].

[20] J. D. Eshelby, Proc. Roy. Soc. London A 241, 376 (1957).

[21] Note that opposite sources (e.g. $\epsilon_{x y}^{\text {core }}= \pm \epsilon_{0}$ in some small zone), give rise (typically) to opposite fields $\pm \epsilon_{x y}^{\text {field }}$ : their contributions to $\left\langle\epsilon_{x y}^{\text {core }}(\underline{r}) \epsilon_{x y}^{\text {field }}(\underline{r}+\underline{R})\right\rangle$ hence add up. This is why the strain-strain correlation picks up the average response to a (positive) shear source even when, at high $T$, shear events are triggered by thermal activation with all possible orientations.

[22] H. Yoshino and M. Mezard, Phys. Rev. Lett. 105, 015504 (Jul. 2010); F. Puosi and D. Leporini, J. of Chem. Phys. 136, 041104 (Jan. 2012).

[23] A. Widmer-Cooper and P. Harrowell, Phys. Rev. Lett. 96, 185701 (May 2006). 